Provided for non-commercial research and education use. Not for reproduction, distribution or commercial use.

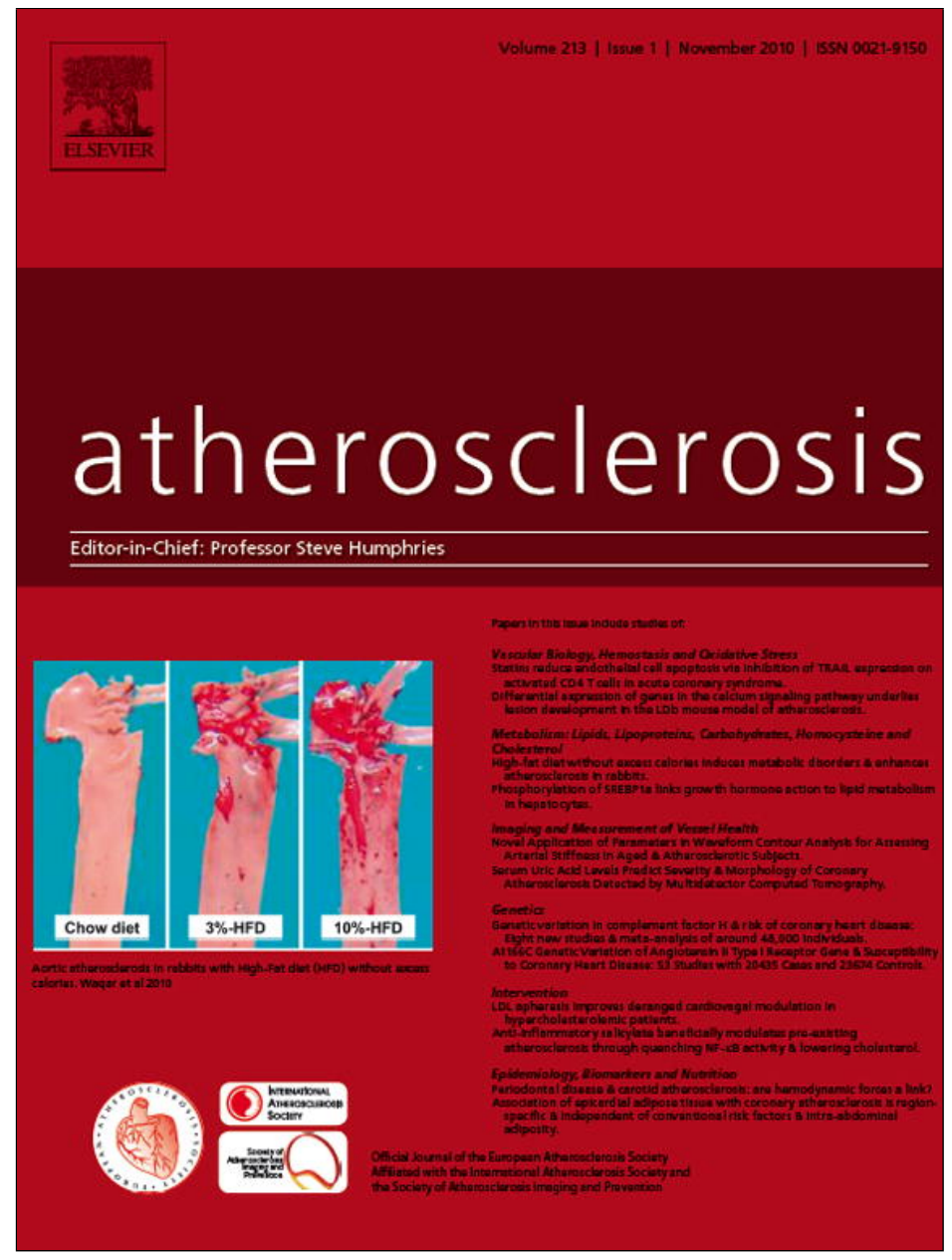

This article appeared in a journal published by Elsevier. The attached copy is furnished to the author for internal non-commercial research and education use, including for instruction at the authors institution and sharing with colleagues.

Other uses, including reproduction and distribution, or selling or licensing copies, or posting to personal, institutional or third party websites are prohibited.

In most cases authors are permitted to post their version of the article (e.g. in Word or Tex form) to their personal website or institutional repository. Authors requiring further information regarding Elsevier's archiving and manuscript policies are encouraged to visit:

http://www.elsevier.com/copyright 


\title{
Immune-inflammatory markers and arterial stiffness indexes in subjects with acute ischemic stroke
}

\author{
Antonino Tuttolomondo*, Domenico Di Raimondo, Rosaria Pecoraro, \\ Antonia Serio, Gisella D’Aguanno, Antonio Pinto, Giuseppe Licata \\ Dipartimento Biomedico di Medicina Interna e Specialistica, Università degli Studi di Palermo, Italy
}

\section{A R T I C L E I N F O}

\section{Article history:}

Received 16 February 2010

Received in revised form 10 July 2010

Accepted 16 August 2010

Available online 26 August 2010

\section{Keywords:}

Stroke

Cytokines

PWV

Aix

\begin{abstract}
A B S T R A C T
No study has yet evaluated the relationship between arterial stiffness indexes and immuno-inflammatory pathway in patients with an acute cardiovascular or cerebrovascular event. The aim of our study was to evaluate in patients with acute ischemic stroke the relationship between immune-inflammatory markers and arterial stiffness indexes.

Methods: 107 subjects with acute ischemic stroke and 107 controls without stroke. We evaluated plasma levels of C-reactive protein (CRP), interleukin-1 beta (IL-1 $\beta$ ), tumor necrosis factor-alpha (TNF- $\alpha$ ), interleukin-6 (IL-6) and interleukin-10 (IL-10), E-selectin, P-selectin, intercellular adhesion molecule-1 (ICAM-1), vascular cell adhesion molecule-1 (VCAM-1), von Willebrand Factor (vWF), tissue plasminogen activator (TPA), plasminogen activator inhibitor-1 (PAI-1). Carotid-femoral pulse wave velocity (PWV) and augmentation index (Aix) were evaluated.

Results: There was a significant positive relationship, corrected for age, and gender, between PWV and CRP, TNF- $\alpha$, IL1 $\beta$, VWF and IL-6. Aix was significantly related to VWF, IL-6 and TNF- $\alpha$ levels. Among Lacunar subtype PWV was significantly related to CRP, IL-1 $\beta$, IL-6, TNF- $\alpha$ and vWF. In LAAS subjects PWV was significantly related to CRP, IL-1 $\beta$, IL-6, TNF- $\alpha$ but not with vWF. Among CEI subtype, PWV was significantly and positively related to CRP, IL- $1 \beta$, TNF- $\alpha$ and vWF.

Discussion: Our findings show that both aortic stiffness and wave reflection are related to the degree of systemic inflammation in stroke subjects, suggesting that circulating inflammation mediators can influence the stiffness of vessels distant to those involved in the disease process itself.
\end{abstract}

(c) 2010 Elsevier Ireland Ltd. All rights reserved.

\section{Introduction}

Inflammation markers such as C-reactive protein (CRP) are predictive of stroke occurrence [1] and CRP has been reported elevated in stroke patients [2], whereas our group [3,4] and other authors $[5,6]$ reported that some pro-inflammatory cytokines are systematically increased after ischemic stroke.

Although there are some inconsistencies, a number of recent studies have suggested that in a healthy population, there may be a significant relationship between CRP and measures of arterial stiffness. Yasmin et al. found CRP to be related to pulse wave velocity (PWV) but not to augmentation index (Aix) [9]. In contrast, Kampus et al. found CRP to be independently and significantly associated with Aix. Moreover in patients with systemic vasculitis, in which

\footnotetext{
* Corresponding author at: Dipartimento Biomedico di Medicina Interna e Specialistica, Università degli Studi di Palermo, P.zza delle Cliniche n.2, 90127 Palermo, Italy. Tel.: +39 091 6552197; fax: +390916552285.

E-mail address: brunotutto@unipa.it (A. Tuttolomondo).
}

CRP levels are markedly elevated, they were positively correlated with PWV and Aix [11].

Arterial stiffness is increasingly recognized as an important determinant of cardiovascular risk [13-16] and may be directly involved in the process of atherosclerosis [17]. The factors underlying increased arterial stiffness are incompletely understood, but both functional and structural alterations in the vessel wall are thought to be important. Indeed, some investigators have shown that endothelial-derived nitric oxide (NO) regulates large artery stiffness in vivo [12,18-21]. This may explain why other cardiovascular risk factors such as diabetes mellitus and hypercholesterolemia, which are associated with endothelial dysfunction, are linked to premature arterial stiffening.

Few studies examined the relationship between arterial stiffness indexes and systemic inflammation markers such as proinflammatory cytokines [22], whereas no study has evaluated the relationship between these indexes and immune-inflammatory markers in patients with an acute cardiovascular or cerebrovascular event. Only one study [23] indirectly evaluated this relationship and only with regard to erythrocyte sedimentation rate (ESR). 
On this basis the aim of our study was to evaluate the relationship between arterial stiffness and cytokine, selectin, adhesion molecule and von Willebrand Factor plasma levels in subjects with acute ischemic stroke.

\section{Methods}

\subsection{Patient selection}

We enrolled all consecutive patients with a diagnosis of acute ischemic stroke admitted to the Internal Medicine Department at the University of Palermo between November 2006 and January 2008 , and hospitalized control patients without a diagnosis of acute ischemic stroke. Control subjects were patients admitted, in the same period, to our Internal Medicine Department, for any cause other than acute cardiovascular and cerebrovascular events.

Stroke was defined by focal neurological signs or symptoms thought to be of vascular origin that persisted for $>24 \mathrm{~h}$ confirmed by brain CT and/or MRI in baseline conditions and brain CT with contrast medium after $48-72 \mathrm{~h}$ [9].

In order to match patients with acute ischemic stroke and controls for cardiovascular risk and previous cardiovascular morbidity, controls were included if they had vascular risk factors or a history of myocardial infarction or cerebrovascular disease or peripheral vascular disease, but they were excluded if they had either current or recent (within 6 months) cerebrovascular disease or one of the exclusion criteria (see above).

Cardiovascular risk factors were evaluated for both cases and controls on the basis of the criteria shown below. Hypercholesterolemia was defined as the presence of total cholesterol blood levels $\geq 200 \mathrm{mg} / \mathrm{dl}$. Hypertension was defined as present if subjects had been previously diagnosed according to the World Health Organization/International Society of Hypertension guidelines and were routinely receiving antihypertensive therapy. Patients were defined as type 2 diabetics if they had known diabetes treated by diet, oral hypoglycaemic drugs or insulin before stroke.

Previous coronary artery disease was determined on the basis of a history of physician-diagnosed angina, myocardial infarction, or any previous revascularization procedure assessed by a questionnaire.

Previous cerebrovascular disease (TIA/ischemic stroke) was assessed by history, specific neurologic examination performed by specialists, and hospital or radiological (brain computer tomography or brain magnetic resonance) records of definite previous stroke.

Subjects were classified as having previous peripheral artery disease (PAD) when they had an history of $\mathrm{ABI}<0.9$ and/or of intermittent claudication or of critical limb ischemia or when they had undergone a peripheral arterial bypass or amputation.

The study protocol was approved by the local ethics committee, and all participants gave written informed consent Every subject with ischemic stroke was matched for age ( \pm 3 years), sex, and cardiovascular risk factor prevalence with one control subject.

The type of acute ischemic stroke was classified according to the TOAST classification [24]: (1) Large Artery AtheroSclerosis (LAAS); (2) CardioEmbolic Infarct (CEI); (3) LACunar infarct (LAC); (4) stroke of Other Determined Etiology (ODE); (5) stroke of UnDetermined Etiology (UDE).

\subsubsection{Large Artery AtheroSclerosis (LAAS)}

These patients have clinical and brain imaging findings of either significant $(>50 \%)$ stenosis or occlusion of a major brain artery or branch cortical artery, presumably due to atherosclerosis. Clinical findings include those of cerebral cortical impairment (aphasia, neglect, restricted motor involvement, etc.) or brain stem or cerebellar dysfunction. Cortical or cerebellar lesions and brain stem or subcortical hemispheric infarcts greater than $1.5 \mathrm{~cm}$ in diameter on CT or MRI are considered to be of potential large-artery atherosclerotic origin. Supportive evidence by duplex imaging or arteriography of a stenosis of greater than $50 \%$ of an appropriate intracranial or extracranial artery is needed. Diagnostic studies should exclude potential sources of cardiogenic embolism.

\subsubsection{CardioEmbolic Infarcts (CEI)}

This category includes patients with arterial occlusions presumably due to an embolus arising in the heart. Cardiac sources are divided into high-risk and medium-risk groups based on the evidence of their relative propensities for embolism. At least one cardiac source for an embolus must be identified for a possible or probable diagnosis of cardioembolic stroke. Clinical and brain imaging findings are similar to those described for large-artery atherosclerosis. Evidence of a previous TIA or stroke in more than one vascular territory or systemic embolism supports a clinical diagnosis of cardiogenic stroke. Potential large-artery atherosclerotic sources of thrombosis or embolism should be eliminated. Stroke in a patient with a medium-risk cardiac source of embolism and no other cause of stroke is classified as a possible cardioembolic stroke.

\subsubsection{LACunar infarct (LAC)}

The patient should have one of the traditional clinical lacunar syndromes and should not have evidence of cerebral cortical dysfunction. A history of diabetes mellitus or hypertension supports the clinical diagnosis. The patient should also have a normal CT/MRI examination or a relevant brain stem or subcortical hemispheric lesion with a diameter of less than $1.5 \mathrm{~cm}$ demonstrated.

\subsubsection{Stroke of Other Determined Etiology (ODE)}

This category includes patients with rare causes of stroke, such as non-atherosclerotic vasculopathies, hypercoagulable states, or hematologic disorders. Patients in this group should have clinical and CT or MRI findings of an acute ischemic stroke, regardless of the size or location. Diagnostic studies such as blood tests or arteriography should reveal one of these unusual causes of stroke. Cardiac sources of embolism and large-artery atherosclerosis should be excluded by other studies.

\subsubsection{Stroke of UnDetermined Etiology (UDE)}

In some cases, the cause of a stroke cannot be determined with any degree of confidence. Some patients have no likely etiology determined despite an extensive evaluation. In others, no cause is found but the evaluation was cursory.

All the ischemic stroke patients underwent: medical history with recording of potential stroke risk factors, blood and coagulation tests, 12-lead ECG, $24 \mathrm{~h}$ electrocardiography monitoring, trans-thoracic echocardiography, carotid ultrasound, brain CT or MRI at admission (repeated between the third and seventh days of stroke onset).

Neurological deficit score on admission was evaluated by Scandinavian Stroke Scale (SSS). SSS assesses neurological deficit through an evaluation of consciousness level, eye movement, strength in arms, hands, and legs, orientation, language, facial weakness and gait, giving rise to a score ranging from 58 (absence of deficit) to 0 (death).

\subsection{PWV measurement}

Carotid-femoral PWV was measured in the supine position using the automatic device (SphygmoCor version 7.1) that measured the time delay between the rapid upstroke of the carotid and femoral artery pulse waves. The distance between the 2 arterial points was measured on the surface of the body using a tape measure. PWV 
was calculated as the distance traveled by the arterial pulse wave (meters) divided by the time delay between the 2 arterial points (seconds), thus expressed as meters per second.

\subsection{Pulse wave analysis}

Applanation tonometry was used to record radial artery pressure waveform continuously, and mean values of $\geq 2$ screens of pulse waves of good quality were used for analysis. On the basis of the collected data, an averaged radial pressure waveform was generated and a corresponding aortic pressure waveform and BP calculated by the validated transfer function (SphygmoCor version 7.1). The aortic pressure waveform was used to calculate the Aix (difference in height between the first and second systolic peaks expressed as a percentage of pulse pressure (PP)).

\subsection{Laboratory evaluation}

Blood samples were obtained in the non-fasting state. After 10 min of rest in the supine position, vital signs were recorded and blood samples were collected from the antecubital vein.

EDTA-anticoagulated peripheral blood was drawn from each patient within $12 \mathrm{~h}$ from symptom onset. Serum and plasma were immediately separated by centrifugation and stored in aliquots at $-80^{\circ} \mathrm{C}$ until analysis.

We evaluated plasma levels of C-reactive protein (CRP), interleukin-1beta (IL-1 $\beta$ ), tumor necrosis factor-alpha (TNF- $\alpha$ ), interleukin-6 (IL-6) and interleukin-10 (IL-10), E-selectin, Pselectin, intercellular adhesion molecule-1 (ICAM-1), vascular cell adhesion molecule-1 (VCAM-1), as markers of immuneinflammatory activation, von Willebrand Factor (vWF) plasma levels as a marker of endothelial dysfunction, tissue plasminogen activator (TPA) and plasminogen activator inhibitor-1 (PAI-1) as marker of thrombotic/fibrinolytic pathway.

IL-1 $\beta$, TNF- $\alpha$, IL- 6 and IL-10 and VWF antigen were measured using a sandwich ELISA (Human IL-1 $\beta$, TNF- $\alpha$, IL-6 and IL-10 Quantikine, R\&D Systems (VWF ELISA kitdurian, Instrumentation Laboratory, Milano, Italy)); VCAM-1, ICAM-1, E-selectin, P-selectin, PAI-1 and TPA-antigen were measured by commercial bioimmunoassay (Human SICAM-1, sVCAM-I, sE-selectin and sP-selectin Parameter, Quantikine, R\&D Systems, Gentaur AssayMax Human Plasminogen Activator Inhibitor-1 (PAI-1) ELISA Kit, Gentaur AssayMax Tissue Plasminogen Activator (TPA) ELISA Kit).

The minimum detectable concentrations for the diagnostic tests are: TNF alpha: $1.6 \mathrm{pg} / \mathrm{ml}$; IL-1 $\beta$ : $<1 \mathrm{pg} / \mathrm{ml}$; IL-6: $<0.70 \mathrm{pg} / \mathrm{ml}$; IL-10: $>3.9 \mathrm{pg} / \mathrm{ml}$; ICAM-1: $<0.35 \mathrm{ng} / \mathrm{ml}$; VCAM-1: $0.6 \mathrm{ng} / \mathrm{ml}$; E-selectin: $<0.1 \mathrm{ng} / \mathrm{ml}$; P-selectin: <0.5 ng/ml; vWF: $1.0 \%$; TPA: 0.3 pg/ml; PAI1: $<50 \mathrm{pg} / \mathrm{ml}$.

Intraassay and interassay coefficients of variation were: TNF alpha: $4.2 \%$ and $4.6 \%$; IL-1 $\beta$ : $3.3 \%$ and $4.2 \%$; IL-6: $1.6 \%$ and $3.3 \%$; IL-10: $4.3 \%$ and $7.5 \%$; ICAM-1: $4.8 \%$ and $6.1 \%$; VCAM-1: $3.5 \%$ and 7.7\%; E-selectin: $4.8 \%$ and 5.7\%; P-selectin: $4.9 \%$ and $8.8 \%$; vWF: $5 \%$ and $10 \%$; TPA: $4.8 \%$ and $5 \%$; PAI-1: $5.7 \%$ and $8.3 \%$.

\subsection{Statistical analysis}

Results are expressed as mean \pm SD for continuous variables and percentages for categorical data, with $P \geq 0.05$ considered significant. Analysis of normality was performed with the Shapiro-Wilk $W$ test. Non-normally distributed data were logarithmically ( $\log 10)$ transformed before analysis.

The relationship between immune-inflammatory markers, PWV, Aix, and other parameters was analyzed using nonparametric methods (Spearman $P$ correlations) after correction for age and gender.
According to sample size calculation a sample size of 100 patient-control pairs had $80 \%$ power at the $5 \%$ significance level to detect a $10 \%$ difference in selected biomarker plasma levels and arterial stiffness indexes between control subjects and patients and between each subtype of stroke.

\section{Results}

We enrolled 107 patients with acute ischemic stroke and 107 control subjects matched for age, sex, cardiovascular risk factors and previous cardiovascular morbidity.

According to the TOAST criteria, the etiology of stroke was large-artery atherosclerosis (LAAS) in 41 (38.31\%) patients, cardioembolism (CEI) in 31 (28.97\%) patients, lacunar stroke in 32 (29.92\%) patients whereas $3(2.80 \%)$ subjects were classified as ODE. Baseline patient characteristics are given in Table 1. Subjects with acute ischemic stroke showed significantly higher plasma levels of CRP, IL-1 $\beta$, IL-6 TNF $\alpha$, E-selectin, P-selectin, I-CAM-1, V-CAM-1, VWF, TPA and PAI-1 (see Table 1). Baseline characteristic of subjects with ischemic stroke with regard of TOAST subtypes are given in Table 2.

PWV and Aix values in stroke patients and in each TOAST subtype are given in Table 1 and in Table 2. Subjects with acute ischemic stroke showed significantly higher levels of PWV and AIx (see Table 1) whereas in relation of stroke subtype lacunar subtype showed the highest value of both arterial stiffness indexes (see Table 2).

\subsection{Relationship between PWV and immune-inflammatory variables}

There was a significant positive relationship, corrected for age, and gender, between PWV and CRP $(r=0.36 ; P<0.001)$, TNF$\alpha(r=0.42 ; P<0.001), \operatorname{IL1} \beta(r=0.35 ; P<0.001), \operatorname{VWF}(r=0.46$; $P<0.001)$, and IL-6 $(r=0.27 ; P<0.05)$ (see Table 3$)$.

\subsection{Relationship between Aix and immune-inflammatory variables}

The Aix was significantly related, after correction for age and gender, to VWF $(r=0.38 ; P<0.0001)$, but not to CRP $(r=0.09$; $P=0.37)$, IL-6 $(r=0.12 ; P<0.08)$, or TNF- $\alpha(r=0.14 ; P<0.15)$ levels (see Table 3).

\subsection{Relationship between PWV and immune-inflammatory variables in relation to TOAST subtype}

Among Lacunar subtype PWV, after correction for age and gender, was significantly and positively related to CRP, IL-1 $\beta$, IL-6, TNF- $\alpha$ and vWF (see Table 4).

In subjects with stroke classified as LAAS, PWV was significantly and positively related to CRP, IL- $1 \beta$, IL-6, TNF- $\alpha$ but not with vWF (see Table 4).

In CEI subtype, PWV was significantly and positively related to TNF- $\alpha$ and vWF but not with CRP, IL-6, IL-1 $\beta$ (see Table 4).

\subsection{Relationship between Aix and immune-inflammatory variables in relation to TOAST subtype}

Among Lacunar and CEI subtype Aix was positively and significantly related, after correction for age and gender, only to vWF; in LAAS subgroup this relationship was significantly weaker and only close to statistical significance (see Table 4). 
Table 1

General characteristics and immuno-inflammatory variables of stroke patients and controls.

\begin{tabular}{|c|c|c|c|}
\hline Variable & Stroke pts ( $n: 107)$ & Controls ( $n: 102)$ & $P$ \\
\hline Age (years) & $71(63-80.5)$ & $68(63-80)$ & 0.79 \\
\hline $\mathrm{M} / \mathrm{F}(n)$ & $66 / 41$ & $57 / 55$ & 0.022 \\
\hline $\mathrm{SBP} / \mathrm{DBP}(\mathrm{mm} / \mathrm{Hg})$ & $151 \pm 8.9 / 96 \pm 4.2$ & $141 \pm 9.8 / 91 \pm 2.2$ & $<0.001$ \\
\hline $\mathrm{MAP}(\mathrm{mm} / \mathrm{Hg})$ & $114.33 \pm 9.4$ & $107.6 \pm 8.4$ & $<0.005$ \\
\hline Diabetes $(n / \%)$ & $44(41.12)$ & $41(40.19 \%)$ & 0.263 \\
\hline Hypertension $(n / \%)$ & $50(45.72 \%)$ & $46(45.09 \%)$ & 0.301 \\
\hline Age (years) & $71(63-80.5)$ & $68(63-80)$ & 0.79 \\
\hline Glucose blood levels (mg/dl) & $148.5(97-213)$ & $109(81.5-163.5)$ & $<0.001$ \\
\hline Cholesterol blood levels (mg/dl) & $231(189-250)$ & $220(168-215)$ & $<0.001$ \\
\hline Triglycerids blood levels (mg/dl) & $177.5(130.75-201.75)$ & $141(96-205)$ & 0.004 \\
\hline White blood cells (per $\mathrm{mm}^{3}$ ) & $9200(6000-13,000)$ & $7400(6500-9800)$ & $<0.001$ \\
\hline Neutrophils (\%) & $6180(5071-9000)$ & $4040(3200-5800)$ & $<0.001$ \\
\hline Diabetes $(n / \%)$ & $44(41.12)$ & $41(40.19 \%)$ & 0.263 \\
\hline Hypertension $(n / \%)$ & $50(45.72 \%)$ & $46(45.09 \%)$ & 0.301 \\
\hline $\operatorname{Aix}(\%)$ & $103 \pm 3.5$ & $89 \pm 4.6$ & $<0.001$ \\
\hline $\mathrm{PWV}(\mathrm{m} / \mathrm{s})$ & $11.8 \pm 3.3$ & $10.02 \pm 2.29$ & $<0.001$ \\
\hline SSS & $30.22 \pm 16.21$ & - & \\
\hline NIHSS & $19.41 \pm 10.06$ & - & \\
\hline \multicolumn{4}{|l|}{ msRankin score at discharge } \\
\hline I & $20(18.69)$ & & \\
\hline II & $22(20.56)$ & & \\
\hline III & $20(18.69)$ & & \\
\hline IV & $24(22.45)$ & & \\
\hline V & $21(19.62)$ & & \\
\hline Death $(n / \%)$ & $7(6.5)$ & - & $<0.001$ \\
\hline $\operatorname{CAD}(n / \%)$ & $38(35.51)$ & $37(36.27)$ & 0.44 \\
\hline $\mathrm{CHF}(n / \%)$ & $21(19.62)$ & $15(14.70)$ & 0.065 \\
\hline Previous TIA (n/\%) & $40(37.38)$ & $11(10.87)$ & $<0.001$ \\
\hline Previous stroke $(n / \%)$ & $33(30.84)$ & $7(6.82)$ & $<0.001$ \\
\hline Microalbuminuria (n/\%) & $41(38.31)$ & $18(17.64)$ & $<0.001$ \\
\hline Carotid plaque ( $n / \%)$ & $61(57)$ & $33(32.35) \mathrm{I}$ & $<0.001$ \\
\hline LVH $(n / \%)$ & $45(42.05)$ & $21(20.58)$ & $<0.001$ \\
\hline $\begin{array}{l}\text { Previous brain infarct at } \\
\text { neuroimage }(n / \%)\end{array}$ & $40(37.38)$ & $19(18.62)$ & $<0.001$ \\
\hline WMHLS $(n / \%)$ & $27(25.23)$ & $18(17.64)$ & 0.024 \\
\hline $\mathrm{CRP}(\mathrm{mg} / \mathrm{dl})$ & $3.8 \pm 2.2$ & $1.9 \pm 0.9$ & $<0.05$ \\
\hline IL-1- $\beta(\mathrm{pg} / \mathrm{ml})$ & $8(5-10)$ & $4(2-5)$ & $<0.001$ \\
\hline IL-6 $(\mathrm{pg} / \mathrm{ml})$ & $10(6-28)$ & $8(3.1-12)$ & $<0.001$ \\
\hline $\mathrm{TNF}-\alpha(\mathrm{pg} / \mathrm{ml})$ & $30.5(10.25-46)$ & $5.1(1.1-4.3)$ & $<0.001$ \\
\hline E-selectin (ng/ml) & $2.05(1.0-3.8)$ & $2(1-2)$ & $<0.001$ \\
\hline P-selectin (ng/ml) & $4.5(2-6.8)$ & $3.1(2.1-4)$ & 0.004 \\
\hline $\operatorname{VICAM}(\mathrm{ng} / \mathrm{ml})$ & $16(10.1-20)$ & $10(7-15)$ & $<0.001$ \\
\hline ICAM $(\mathrm{ng} / \mathrm{ml})$ & $18.8(12.2-20)$ & $10.9(12-16.1)$ & $<0.001$ \\
\hline $\mathrm{IL}-10(\mathrm{pg} / \mathrm{ml})$ & $3.95(2-7)$ & $4(2-10)$ & 0.233 \\
\hline $\operatorname{vWF}(\mathrm{ng} / \mathrm{ml})$ & $11(6-15)$ & $4(3-9)$ & 0.0001 \\
\hline PAI- 1 (pg/ml) & $137(99.5-155)$ & $23(11-24)$ & $<0.001$ \\
\hline $\mathrm{TPA}(\mathrm{pg} / \mathrm{ml})$ & $21(10.55-39)$ & $55(29-88)$ & $<0.001$ \\
\hline \multicolumn{4}{|l|}{ Premorbid antithrombotics } \\
\hline Antiplatelets $(n / \%)$ & $41(38.31)$ & $34(33.33)$ & 0.74 \\
\hline Anticoagulants $(n / \%)$ & $33(30.84)$ & $27(26.47)$ & 0.54 \\
\hline \multicolumn{4}{|l|}{ Premorbid cardiovascular drugs } \\
\hline Ace-inhibitors $(n / \%)$ & $39(36.44)$ & $30(29.41)$ & 0.041 \\
\hline ARBs $(n / \%)$ & $28(26.18)$ & $21(20.58)$ & 0.40 \\
\hline Statins $(n / \%)$ & $42(39.25)$ & $32(31.37)$ & 0.35 \\
\hline Antidiabetic drugs ( $n / \%)$ & $21(19.62)$ & $22(21.50)$ & 0.21 \\
\hline Biguanids $(n / \%)$ & $12(11.21)$ & $14(13.72)$ & 0.35 \\
\hline Sulphonilureas (n/\%) & $9(8.41)$ & $8(7.8)$ & 0.67 \\
\hline Thiazolidinediones ( $n / \%)$ & - & - & \\
\hline Insulin $(n / \%)$ & $23(21.49)$ & 19 & 0.28 \\
\hline
\end{tabular}

SBP: systolic blood pressure; DBP: diastolic blood pressure; MAP: mean arterial pressure; Aix: augmentation index; PWV: pulse wave velocity; SSS: Scandinavian Stroke Scale score; NIHSS: National Institutes of Health Stroke Scale; Angiotensin II receptor blockers (ARBs); CAD: coronary artery disease; CHF: congestive heart failure; LVH: left ventricular hypertrophy; WMHLS: white matter hyperintensity lesions; TIA: transitory ischemic attack; CRP: C-reactive protein; TNF- $\alpha$ : tumor necrosis factor $\alpha$; IL-1 $\beta$ : interleukin-1- $\beta$; IL-6: interleukin-6; IL-10: interleukin 10; ICAM-1: intercellular adhesion molecule-1; VCAM-1: vascular cell adhesion molecule-1; vWF: von Willebrand Factor; TPA: tissue plasminogen activator; PAI-1: plasminogen activator inhibitor-1.

Immuno-inflammatory and thrombotic-fibrinolytic variables are expressed as median and interquartile (lower and upper quartile).

Demographic and anamnestic data are expressed as no. (percentage).

\section{Discussion}

The main our findings were that patients with acute ischemic stroke had both increased inflammatory markers and arterial stiffness markers compared to control subjects without acute ischemic stroke.
This is the first study to show in patients with acute ischemic stroke that circulating levels of some immune-inflammatory markers such as CRP, IL-6, IL- $1 \beta$, TNF- $\alpha$ and VWF are related to PWV and wave reflection.

Large artery stiffness is now recognized as a modifiable, independent predictor of cardiovascular risk [26,27]. Structural 
Table 2

Premorbid cardiovascular risk factors, clinical characteristics and antithrombotic medication by ischemic stroke subtype.

\begin{tabular}{|c|c|c|c|c|c|}
\hline Variable & Lacunar & LAAS & CEI & ODE & $P$ \\
\hline Number & 32 & 41 & 31 & 3 & \\
\hline Age (years) & $76.31 \pm 7.37$ & $72.17 \pm 6.83$ & $76.00 \pm 8.79$ & $49.00 \pm 45.50$ & $<0.001$ \\
\hline $\operatorname{Sex}(M / F)$ & $19 / 12$ & $29 / 12$ & $23 / 8$ & $4 / 0$ & $<0.001$ \\
\hline Diabetes $(n / \%)$ & $17(56.25)$ & $18(43.90)$ & $9(29.03)$ & 0 & $<0.001$ \\
\hline Hypertension $(n / \%)$ & $18(56.2)$ & $17(41.46)$ & $14(45.16)$ & $1(33.33)$ & 0.25 \\
\hline Hypercholesterolaemia ( $n / \%)$ & $7(21.87)$ & $16(39.02)$ & $9(29.03)$ & 0 & $<0.05$ \\
\hline Atrial fibrillation $(n / \%)$ & $3(9.3)$ & $5(12.19)$ & $24(77.41)$ & 0 & $<0.001$ \\
\hline Previous TIA $(n / \%)$ & $5(15.6)$ & $13(31.07)$ & $10(32.2)$ & $1(33.3 \%)$ & $<0.001$ \\
\hline Previous stroke $(n / \%)$ & $7(21.8)$ & $8(19.5)$ & $7(22.05)$ & $2(66.66)$ & 0.038 \\
\hline Glucose blood levels (mg/dl) & $169.12 \pm 74.06$ & $117.00 \pm 53.44$ & $102.29 \pm 7.68$ & $92.29 \pm 17.68$ & $<0.001$ \\
\hline Total cholesterol blood levels (mg/dl) & $179.56 \pm 40.07$ & $175.02 \pm 46.42$ & $165.71 \pm 30.64$ & $169.00 \pm 49.46$ & $<0.05$ \\
\hline LDL cholesterol $(\mathrm{mg} / \mathrm{dl})$ & $95.62 \pm 38.65$ & $103.247 \pm 28.9$ & $84.67 \pm 33.99$ & $99.20 \pm 32.81$ & $<0.05$ \\
\hline Trygliceride blood levels (mg/dl) & $170.16 \pm 82.40$ & $190.82 \pm 162.12$ & $112.35 \pm 32.36$ & $148.00 \pm 83.439$ & $<0.05$ \\
\hline WBC $\left(\right.$ per $\left.\mathrm{mm}^{3}\right)$ & $10041.37 \pm 4167.31$ & $11742.82 \pm 9267.20$ & $16282.41 \pm 2556.64$ & $10785.00 \pm 5437.65$ & $<0.05$ \\
\hline Neutrophile (\%) & $7384.51 \pm 3769.68$ & $7803.87 \pm 4303.00$ & $6996.13 \pm 3107.86$ & $8418.00 \pm 7481.19$ & $<0.05$ \\
\hline HCT $(\%)$ & $39.20 \pm 3.9$ & $40.70 \pm 4.24$ & $41.386 \pm 3.196$ & $38.25 \pm 9.54$ & 0.041 \\
\hline SB P/DBP (mm/Hg) & $153 \pm 9.5 / 96 \pm 4.2$ & $151 \pm 6.5 / 95 \pm 4.2$ & $149 \pm 9.8 / 93 \pm 2.2$ & $145 \pm 5.8 / 91 \pm 2.5$ & $<0.05$ \\
\hline $\mathrm{MAP}(\mathrm{mm} / \mathrm{Hg})$ & $115 \pm 9.7$ & $113.6 \pm 10.1$ & $111.6 \pm 8.2$ & $109 \pm 6.7$ & $<0.05$ \\
\hline Aix $(\%)$ & $112 \pm 5.5$ & $108 \pm 3.5$ & $99 \pm 3.5$ & $101 \pm 3.5$ & $<0.05$ \\
\hline $\mathrm{PWV}(\mathrm{m} / \mathrm{s})$ & $12.04 \pm 1.54$ & $11.98 \pm 2.445$ & $10.40 \pm 2.445$ & $11.48 \pm 1.345$ & $<0.05$ \\
\hline NIHSS & $14.65 \pm 14.59$ & $20.51 \pm 16.06$ & $15.64 \pm 8.73$ & $19.00 \pm 12.72$ & $<0.05$ \\
\hline \multicolumn{6}{|l|}{ msRankin score at discharge ( $n / \%)$} \\
\hline I & $8(25)$ & $7(17.07)$ & $4(12.90)$ & $1(25)$ & \\
\hline II & $13(18.75)$ & $6(14.63)$ & $3(9.6)$ & - & \\
\hline III & $6(40.62)$ & $9(21.95)$ & $5(16.19)$ & $2(50)$ & \\
\hline IV & $3(9.37)$ & $10(24.39)$ & $10(32.22)$ & $1(25)$ & \\
\hline V & $2(6.25)$ & $9(21.95)$ & $9(29.03)$ & - & \\
\hline Death $(n / \%)$ & - & $2(4.8)$ & $5(16.12)$ & - & \\
\hline $\operatorname{CAD}(n / \%)$ & $13(40.6)$ & $18(43.91)$ & $7(22.58)$ & 0 & \\
\hline $\mathrm{CHF}(n / \%)$ & $4(12.5)$ & $5(12.19)$ & $11(35.48)$ & $1(33.33)$ & \\
\hline Microalbuminuria ( $n / \%)$ & $24(75 \%)$ & $11(26.82)$ & $6(19.35)$ & 0 & $<0.001$ \\
\hline Carotid plaque $(n / \%)$ & $22(68.7)$ & $27(65.85)$ & $14(45.16)$ & 0 & $<0.05$ \\
\hline $\operatorname{LVH}(n / \%)$ & $18(56.25)$ & $17(41.46)$ & $10(32.25)$ & 0 & $<0.05$ \\
\hline $\begin{array}{l}\text { Previous brain infarct at } \\
\text { neuroimaging }(n / \%)\end{array}$ & $13(40.62)$ & $15(36.58)$ & $10(32.2)$ & $2(66.66)$ & 0.65 \\
\hline WMHLS $(n / \%)$ & $13(40.6)$ & $8(25.80)$ & $5(16.1)$ & $1(66.66)$ & $<0.05$ \\
\hline $\mathrm{CRP}(\mathrm{mg} / \mathrm{dl})$ & $2.8 \pm 2.1$ & $3.9 \pm$ & $3.1 \pm 1.7$ & $1.8 \pm 0.9$ & 0.041 \\
\hline $\mathrm{IL}-1-\beta(\mathrm{pg} / \mathrm{ml})$ & $5(4-9)$ & $8(6-11)$ & $11(6-12)$ & $7(5-11)$ & $<0.05$ \\
\hline IL-6 (pg/ml) & $8(6-21)$ & $10(5-25)$ & $12(6-29)$ & $10(6-28)$ & $<0.05$ \\
\hline $\mathrm{TNF}-\alpha(\mathrm{pg} / \mathrm{ml})$ & $22.5(8.5-26)$ & $30.5(11.25-40)$ & $39.5(24-55)$ & $32.5(10.25-46)$ & $<0.05$ \\
\hline E-selectin (ng/ml) & $3.05(1.5-3.7)$ & $2.45(1.6-3.9)$ & $3.05(2.0-4.0)$ & $2.05(1.0-3.8)$ & 0.035 \\
\hline P-selectin (ng/ml) & $3.5(2-4.8)$ & $4.4(2-6.0)$ & $4.6(2-6.9)$ & $4.5(2-6.8)$ & 0.71 \\
\hline VICAM (ng/ml) & $16(9.1-18)$ & $14(10.2-21)$ & $17(10.1-22)$ & $16(10.51-20)$ & 0.67 \\
\hline ICAM (ng/ml) & $16.8(11.2-18)$ & $17.9(10.2-210)$ & $20.6(11.2-24)$ & $17.8(13.2-21)$ & 0.041 \\
\hline $\mathrm{IL}-10(\mathrm{pg} / \mathrm{ml})$ & $3.55(2-6)$ & $3.96(2-8)$ & $3.2(2-9)$ & $3.95(2-7)$ & 0.65 \\
\hline $\mathrm{vWF}(\mathrm{ng} / \mathrm{ml})$ & $10(4-17)$ & $12(5-13)$ & $14(6-18)$ & $11(7-116)$ & 0.021 \\
\hline PAI-1 (pg/ml) & $139(90.5-143)$ & $138(91.5-135)$ & $139(99.0-145)$ & $137(99.5-155)$ & 0.34 \\
\hline $\mathrm{TPA}(\mathrm{pg} / \mathrm{ml})$ & $23(10.15-37)$ & $23(10.45-41)$ & $24(12.1-47)$ & $22(10.55-39)$ & 0.81 \\
\hline \multicolumn{6}{|l|}{ Premorbid antithrombotics } \\
\hline Antiplatelets ( $n / \%)$ & $11(38.31)$ & $20(48.78)$ & $10(32.22)$ & - & $<0.05$ \\
\hline Anticoagulants $(n / \%)$ & $4(12.5)$ & $7(17.07)$ & $21(67.74)$ & 1 & $<0.001$ \\
\hline \multicolumn{6}{|l|}{ Premorbid cardiovascular drugs } \\
\hline Ace-inhibitors $(n / \%)$ & $18(56.25)$ & $11(26.82)$ & $10(32.25)$ & - & $<0.05$ \\
\hline ARBs $(n / \%)$ & $10(31.25)$ & $11(26.82)$ & $7(22.58)$ & - & 0.041 \\
\hline Statins $(n / \%)$ & $15(46.87)$ & $20(48.78)$ & $7(22.58)$ & & 0.031 \\
\hline \multicolumn{6}{|l|}{ Antidiabetic drugs ( $n / \%)$} \\
\hline Biguanids $(n / \%)$ & $10(31.25)$ & $7(17.07)$ & $4(12.9)$ & - & $<0.05$ \\
\hline Sulphonilureas $(n / \%)$ & $6(18.75)$ & $3(7.31)$ & $2(6.45)$ & & $<0.05$ \\
\hline Thiazolidinediones ( $n / \%)$ & $4(12.5)$ & $4(9.7)$ & $2(6.45)$ & & $<0.025$ \\
\hline Insulin $(n / \%)$ & $11(34.37)$ & $6(14.63)$ & $6(19.35)$ & - & $<0.05$ \\
\hline
\end{tabular}

SBP: systolic blood pressure; DBP: diastolic blood pressure; MAP: mean arterial pressure; Aix: augmentation index; PWV: pulse wave velocity; SSS: Scandinavian Stroke Scale score; NIHSS: National Institutes of Health Stroke Scale; Angiotensin II receptor blockers (ARBs); CAD: coronary artery disease; CHF: congestive heart failure; LVH: left ventricular hypertrophy; WMHLS: white matter hyperintensity lesions; CRP: C-reactive protein; TNF- $\alpha$ : tumor necrosis factor $\alpha$; IL-1 $\beta$ : interleukin-1- $\beta$; IL-6: interleukin6; IL-10: interleukin 10; ICAM-1: intercellular adhesion molecule-1; VCAM-1: vascular cell adhesion molecule-1; vWF: von Willebrand Factor; TPA: tissue plasminogen activator; PAI-1: PAI-1: plasminogen activator inhibitor-1.

Immuno-inflammatory and thrombotic-fibrinolytic variables are expressed as median and interquartile (lower and upper quartile).

Demographic and anamnestic data are expressed as no. (percentage).

Significative $R$ and $P$ are in bold.

components within the arterial wall, mainly collagen and elastin, together with transmural pressure, are key determinants of large vessel stiffness. However, smooth muscle tone also influences the stiffness of elastic and muscular arteries, suggesting functional regulation of stiffness by local and/or circulating vasoactive substances
[28-30]. Interestingly, acute systemic inflammation has recently been associated with endothelial dysfunction in vivo [31-33].

Our findings show that both aortic stiffness and wave reflection are related to the degree of systemic inflammation in stroke subjects, suggesting that circulating inflammation mediators such 
Table 3

Correlations of pulse wave velocity (PWV) and augmentation index (Aix) with immuno-inflammatory variables in stroke patients.

\begin{tabular}{|c|c|c|c|c|}
\hline \multirow[t]{2}{*}{ Variable } & \multicolumn{2}{|c|}{ Pulse wave velocity } & \multicolumn{2}{|c|}{ Augmentation index (Aix) } \\
\hline & $R$ & $P$-values & $R$ & $P$-values \\
\hline CRP & 0.36 & $<0.001$ & 0.09 & 0.37 \\
\hline IL- $1-\beta$ & 0.35 & $<0.001$ & 0.10 & 0.22 \\
\hline IL-6 & 0.27 & $<0.05$ & 0.12 & 0.35 \\
\hline TNF- $\alpha$ & 0.42 & $<0.001$ & 0.14 & 0.15 \\
\hline E-selectin & 0.13 & 0.42 & 0.12 & 0.22 \\
\hline P-selectin & 0.11 & 0.56 & 0.12 & 0.35 \\
\hline VICAM-1 & 0.08 & 0.37 & 0.08 & 0.37 \\
\hline ICAM-1 & 0.10 & 0.7 & 0.10 & 0.7 \\
\hline IL-10 & 0.11 & 0.81 & 0.10 & 0.77 \\
\hline vWF & 0.46 & $<0.001$ & 0.38 & $<0.0001$ \\
\hline PAI-1 & 0.12 & 0.42 & 0.13 & 0.32 \\
\hline TPA & 0.15 & 0.42 & 0.14 & 0.36 \\
\hline
\end{tabular}

Coefficients $(R)$ and $P$-values are calculated by the Pearson correlation mode. CRP: C-reactive protein; TNF- $\alpha$ : tumor necrosis factor $\alpha$; IL-1 $\beta$ : interleukin-1- $\beta$; IL-6: interleukin-6; IL-10: interleukin 10; ICAM-1: intercellular adhesion molecule1; VCAM-1: vascular cell adhesion molecule-1; vWF: von Willebrand Factor; TPA: tissue plasminogen activator; PAI-1: PAI-1: plasminogen activator inhibitor-1. Significative $R$ and $P$ are in bold.

as CRP and some pro-inflammatory cytokines can influence the stiffness of vessels distant to those involved in the disease process itself.

Previous studies in healthy subjects have shown a positive relationship between either PWV or Aix with CRP but not with both [9,10], whereas Mahmud and Feely [22] showed in hypertensive patients a relationship between augmentation index to IL- 6 and TNF- $\alpha$.

PWV is a classic marker of aortic stiffness, whereas Aix is far more complex and is composed of the magnitude of arterial wave reflection, PWV, and pattern of left ventricular ejection. Also, the determinants of these indices differ. Aix is influenced to a greater extent by the level of heart rate, BP, gender, age, and height, whereas PWV is predominantly determined by age and BP [25].

Aix and PWV represent not only large artery stiffness but, very importantly, the vascular smooth muscle tone in the peripheral medium-sized muscular arteries, emphasizing the systemic nature of the inflammatory response encompassing biochemical and hemodynamic parameters. Cytokine plasma levels in its turn also influences vascular vulnerability to the inflammatory response and decreased production of the endogenous vasodilator nitric oxide (NO) [34]. Inhibition of basal NO synthesis increases aortic Aix and velocity in vivo [35].
An immune-inflammatory cascade occurs after an acute ischemic stroke $[5,6]$ so on this basis cytokine levels that we reported and measured $72 \mathrm{~h}$ from symptom onset express an acute rise of these inflammatory markers.

Our group [3,4] recently showed some differences in acute immune-inflammatory activation with regard of plasma levels of inflammatory markers between each diagnostic subtype of stroke.

We also evaluated the predictive value of a series of candidate serum immuno-inflammatory and thrombotic/fibrinolitic molecules towards diagnosis of acute ischemic stroke showing that TNF- $\alpha$, PAI- 1 and TPA on bivariate logistic regression were highly correlated to stroke diagnosis [7].

Furthermore [8] we recently reported that patients with acute ischemic stroke in comparison with patients without stroke show higher arterial stiffness index values and that among stroke patients, lacunar subtype has the highest arterial stiffness indexes.

On this basis it's possible to hypothesize a possible relationship between acute immuno-inflammatory cascade and acute arterial stiffness increase in patients with acute ischemic stroke and our findings concerning a relationship between some cytokines plasma levels and PWV and Aix may represent a possible confirmation of this association.

What is the relationship between acute high levels of cytokines and arterial stiffness indexes in stroke patients? Does a hyper-acute inflammatory state after ischemic stroke directly cause increase of arterial stiffness? Or in stroke-prone patients with a chronic inflammatory activation exacerbated by acute stressor events such an acute cerebrovascular event, does it represent the pathogenetic basis of high arterial stiffness?

It's difficult to answer; nevertheless, it's possible to obtain an answer to the question about the relationship between inflammation markers and arterial stiffness indexes in acute ischemic stroke by evaluating our findings of some significant correlations at intragroup analysis in each TOAST subtype between arterial stiffness indexes and immune-inflammatory markers.

For instance, only in lacunar subtype we observed a strong correlation between PWV and IL6, IL-1 $\beta$, TNF- $\alpha$ and vWF, whereas in LAAS we did not report any correlation with vWF and in the CEI group we reported only a positive relation with TNF- $\alpha$ and vWF.

Moreover evidence has shown that platelet reactivity is higher in patients following ischemic stroke [34,36]. The correlation between vWF and arterial stiffness markers does not indicate a direct biological background although the possible inflammatory role recently demonstrated for $\mathrm{VWF}[35,37]$, could explain the relationship with arterial stiffness indexes.

Table 4

Correlations of pulse wave velocity (PWv) and augmentation index (Aix) with immuno-inflammatory variables in stroke patients in relation of TOAST stroke subtype.

\begin{tabular}{|c|c|c|c|c|c|c|c|c|c|c|c|c|}
\hline \multirow[t]{3}{*}{ Variables } & \multicolumn{4}{|c|}{ Lacunar } & \multicolumn{4}{|c|}{ LAAS } & \multicolumn{4}{|l|}{ CEI } \\
\hline & \multicolumn{2}{|l|}{ PWV } & \multicolumn{2}{|l|}{ Aix } & \multicolumn{2}{|l|}{ PWV } & \multicolumn{2}{|l|}{ Aix } & \multicolumn{2}{|l|}{ PWV } & \multicolumn{2}{|l|}{ Aix } \\
\hline & $R$ & $P$ & $R$ & $P$ & $R$ & $P$ & $R$ & $P$ & $R$ & $P$ & $R$ & $P$ \\
\hline CRP & 0.37 & $<0.001$ & 0.022 & 0.19 & 0.35 & $<0.001$ & 0.33 & 0.022 & 0.22 & 0.06 & 0.12 & 0.78 \\
\hline IL- $1-\beta$ & 0.272 & $<0.05$ & 0.25 & 0.121 & 0.32 & $<0.05$ & 0.11 & 0.27 & 0.20 & 0.07 & 0.18 & 0.980 \\
\hline IL-6 & 0.27 & $<0.05$ & 0.12 & 0.10 & 0.29 & $<0.05$ & 0.12 & 0.022 & 0.19 & 0.567 & 0.12 & 0.78 \\
\hline TNF- $\alpha$ & 0.36 & $<0.001$ & 0.11 & 0.121 & 0.35 & $<0.001$ & 0.10 & 0.27 & 0.34 & $<0.001$ & 0.18 & 0.980 \\
\hline E-selectin & 0.022 & 0.19 & 0.08 & 0.11 & 0.15 & 0.27 & 0.13 & 0.10 & 0.16 & 0.267 & 0.12 & 0.78 \\
\hline P-selectin & 0.25 & 0.121 & 0.09 & 0.12 & 0.12 & 0.022 & 0.12 & 0.11 & 0.18 & 0.98 & 0.18 & 0.980 \\
\hline VICAM & 0.10 & 0.19 & 0.70 & 0.07 & 0.15 & 0.27 & 0.12 & 011 & 0.18 & 0.98 & 0.14 & 0.68 \\
\hline ICAM & 0.09 & 0.10 & 0.014 & 0.19 & 0.14 & 0.48 & 0.33 & 0.022 & 0.0367 & 0.12 & 0.10 & 0.788 \\
\hline IL-10 & 0.11 & 0.121 & 0.12 & 0.121 & 0.25 & $<0.05$ & 0.19 & 0.27 & 0.19 & 0.32 & 0.12 & 0.78 \\
\hline vWF & 0.39 & $<0.001$ & 0.38 & $<0.001$ & 0.21 & 0.065 & 0.20 & 0.06 & 0.39 & $<0.001$ & 0.37 & $<0.001$ \\
\hline PAI-1 & 0.11 & 0.19 & 0.12 & 0.20 & 0.13 & 0.21 & 0.08 & 0.78 & 0.022 & 0.19 & 0.12 & 0.78 \\
\hline TPA & 0.12 & 0.20 & 0.13 & 0.21 & 0.14 & 0.22 & 0.12 & 0.20 & 0.11 & 0.19 & 0.132 & 0.980 \\
\hline
\end{tabular}

Coefficients $(R)$ and $P$-values are calculated by the Pearson correlation mode.

CRP: C-reactive protein; TNF- $\alpha$ : tumor necrosis factor $\alpha$; IL-1 $\beta$ : interleukin-1- $\beta$; IL-6: interleukin-6; IL-10: interleukin 10; ICAM-1: intercellular adhesion molecule-1; VCAM1: vascular cell adhesion molecule-1; vWF: von Willebrand Factor; TPA: tissue plasminogen activator; PAI-1: PAI-1: plasminogen activator inhibitor-1.

Significative $R$ and $P$ are in bold. 
On this basis it is possible that observed differences with regard to the relationship between arterial stiffness indexes and inflammatory markers could be related to differences in immuneinflammatory activation of the acute phase of each TOAST diagnostic subtype.

Cardioembolic stroke could appear as the "less atherosclerotic" among each diagnostic subtype of stroke and this finding could explain the correlation only with CRP and vWF, although our group recently demonstrated that this subtype of acute ischemic stroke is characterized by the highest degree of immune-inflammatory activation of the acute phase [38].

Our finding concerning some differences in the observed correlation between immune-inflammatory markers and arterial stiffness indexes in relation to each TOAST subtype of stroke might also indicate a different degree of vascular anatomy impairment or a different distribution of certain risk factors already reported as related to the inflammation markers, such as hypertension and diabetes. In fact our patients with lacunar stroke, in which both CRP, IL-6, IL-1 $\beta$, TNF- $\alpha$ and vWF are related to PWV, have an higher frequency of both hypertension and diabetes and this finding allow it to represent, in some ways the prototype of the acute cerebrovascular event in diabetic or hypertensive subjects. Furthermore the molecular events associated with remodelling of the large and medium to small arteries also have been well characterized and involve the combinatorial influences of adhesion molecules, integrins, metallo-proteinases, the renin-angiotensin axis, and inflammation on the cellular constituents (endothelial cells, vascular smooth cells, fibroblasts, and matrix components) of the vasculature $[39,40,42]$.

Biomarkers of inflammation like CRP, interleukin-6, and tumor necrosis factor- $\alpha$ have been associated positively with both indirect (e.g. brachial artery pulse pressure) [41] and direct measures of arterial stiffness in previous studies in apparently healthy individuals, [9] in specific patient groups (e.g. patients with hypertension) [22], and in community-based samples [43-45]. Most of these studies had modest sample sizes, but no study evaluated the relationship between stiffness indexes and immune-inflammatory markers in patients with acute ischemic stroke or other acute cardiovascular event, so our findings appear novel.

A number of possible confounding factors (including additional cardiovascular risk factors, smoking, and other clinical variables as well as pharmacological and non pharmacological treatments) might negatively confound our observed findings. Nevertheless, we compared our patients with acute ischemic stroke patients with matched controls for cardiovascular risk factor. Moreover, we observed no significant difference between case and controls with regard of treatment with ace-inhibitors, ARBs, statins and antidiabetic drugs, potentially able to interfere with vascular inflammation and arterial stiffness markers.

Only one study examined the relationship between arterial stiffness markers and an inflammation marker in subjects with acute ischemic stroke [23].

These authors studied the relationship of arterial stiffness, measured by carotid-femoral pulse wave velocity and inflammation, measured by serum erythrocyte sedimentation rate among 334 ischemic stroke patients. There was a significant correlation between carotid-femoral pulse wave velocity and erythrocyte sedimentation rate $(P=0.001)$, a relationship independent of age, hypertension, diabetes and smoking.

The same authors examined the relationship also investigated the role of inflammation measured by serum erythrocyte sedimentation rate (ESR) in the metabolic syndrome-arterial stiffness relationship amongst 229 prospectively recruited acute ischemic stroke patients, we measured carotid-femoral PWV using applanation tonometry and the inflammatory marker serum ESR. They showed that Carotid-femoral PWV was significantly higher amongst patients with MetS ( $P$, increased waist circumference, raised blood pressure and abnormal glycemia and increased with the number of MetS components).

Our study is the first study that analyzed in patients with acute cerebrovascular events, the relationship between markers of immuno-inflammatory activation such as plasma levels of proinflammatory cytokines and both markers of arterial stiffness such as pulse wave velocity (PWV) and augmentation index (Aix) and thus providing a possible explanation for increased arterial stiffness in patients with acute ischemic stroke [46].

Possible limitations of our study are that our findings corroborate previous observational data showing an association between arterial stiffness and inflammatory markers but it does not provide an explanatory causative link for these associations. Previous studies have shown that exogenously administered cytokines (specifically IL-1 $\beta$ ) cause a NO-mediated basal vasodilation in human veins by inducing the constitutively expressed endothelial nitric oxide (NO)-synthase [45]. Nevertheless in acute ischemic stroke excitotoxic or ischemic conditions excessively activate nNOS, resulting in concentrations of NO that are toxic to surrounding neurons [47]. But what are extra-cerebral large artery levels of NO in ischemic stroke subjects? No study to our knowledge analyzed this issue.

Another possible limitation because of technical limitations with regard of Aix evaluation is that we recruited in patients with cardioembolic subtype of stroke patients subjects with atrial fibrillation. However, other studies that have assessed the markers of arterial stiffness in patients with atrial fibrillation, have evaluated both PWV and Aix $[48,49]$.

Maybe an acute NO depletion role is not directly presumable to explain an acute rise in arterial stiffness in acute stroke patients. For this series of limitations whether our findings can be extended to a chronic situation is a matter of discussion that future studies should be clarify evaluating arterial stiffness indexes change in subjects at cerebrovascular risk before and after an acute ischemic cerebrovascular event.

The arterial changes have a multitude of potential interconnected causes including endothelial dysfunction, oxidative stress, inflammation, atherosclerosis and vascular calcification. The role and contribution of the biochemical changes to arterial stiffness in the acute phase ischemic stroke is not known, but it's likely that immune-inflammatory activation, acute hyperglycemia, endothelial dysfunction and other toxic effects could be responsible of a acute increase of arterial stiffness after ischemic stroke.

This finding may have potential therapeutic applications aimed to reducing arterial stiffness by interfering with these biochemical changes of acute phase after ischemic stroke by means neuroprotective agents like anti-inflammatory or anti-oxidizing agents or cardiovascular drug with potential pleiotropic actions such as statins.

In conclusion we reported that both aortic stiffness and wave reflection are related to the degree of systemic inflammation in stroke subjects, showing a significant relationship between immuno-inflammatory marker and PWV in LAAS and lacunar subtype of stroke and suggesting that circulating inflammation mediators after acute ischemic stroke can influence the stiffness of vessels distant to those involved in the disease process itself.

\section{Conflict of interest}

All the authors state that they have no conflicts to disclose.

\section{References}

[1] Rost NS, Wolf PA, Kase CS, et al. Plasma concentration of C-reactive protein and risk of ischemic stroke and transient ischemic attack: the Framingham study. Stroke 2001;32:2575-9. 
[2] Modrego PJ, Boned B, Berlanga JJ, Serrano M. Plasmatic B-type natriuretic peptide and C-reactive protein in hyperacute stroke as markers of CT-evidence of brain edema. Int J Med Sci 2008;5(January (1)):18-23.

[3] Licata G, Tuttolomondo A, Corrao S, et al. Immunoinflammatory activation during the acute phase of lacunar and non-lacunar ischemic stroke: association with time of onset and diabetic state. Int J Immunopathol Pharmacol 2006;19(July-September (3)):639-46.

[4] Licata G, Tuttolomondo A, Di Raimondo D, et al. Immunoinflammatory activation in acute cardioembolic stroke in comparison with other subtypes of ischemic stroke. Thromb Haemost 2008;101(5):929-37.

[5] Zoppo G, Ginis I, Hallenback JM, et al. Inflammation and stroke: putative role for cytokines, adhesion molecules and i-NOS in brain response to ischemia. Brain Pathol 2000;10:95-101.

[6] Sotgiu S, Zanda B, Marchetti B, et al. Inflammatory biomarkers in blood of patients with acute brain ischemia. Eur J Neurol 2006;13(May (5)):505-13.

[7] Tuttolomondo A, Pinto A, Corrao S, et al. Immuno-inflammatory and thrombotic/fibrinolytic variables associated with acute ischemic stroke diagnosis. Atherosclerosis 2009;203(April (2)):503-8.

[8] Tuttolomondo A, Di Sciacca R, Di Raimondo D, et al. Arterial stiffness indexes in acute ischemic stroke: relationship with stroke subtype. Atherosclerosis 2010;(February 18) [Epub ahead of print].

[9] Yasmin, McEniery CM, Wallace S, et al. C-reactive protein is associated with arterial stiffness in apparently healthy individuals. Arterioscler Thromb Vasc Biol 2004;24:969-74.

[10] Kampus R, Kals J, Ristimãe T, et al. High sensitivity C-reactive protein affects central hemodynamics and augmentation index in apparently healthy persons. J Hypertens 2005;22:1133-9.

[11] Booth $A D$, Wallace $S$, McEniery CM, et al. Inflammation and arterial stiffnes in systemic vasculitis. A model of vascular inflammation. Arthritis Rheum 2004;50:581-8.

[12] Vaitkevicius PV, Fleg JL, Engel JH, et al. Effects of age and aerobic capacity on arterial stiffness in healthy adults. Circulation 1993;88:1456-62.

[13] Boutouyrie P, Tropeano AI, Asmar R, et al. Aortic stiffness is an independen predictor of primary coronary events in hypertensive patients: a longitudinal study. Hypertension 2002;39:10-5.

[14] Lehmann ED, Watts GF, Gosling RG. Aortic distensibility and hypercholesterolaemia. Lancet 1992;340:1171-2.

[15] Lehmann ED, Gosling RG, Sonksen PH. Arterial wall compliance in diabetes. Diabet Med 1992;9:114-9.

[16] Blacher J, Guerin AP, Pannier B, et al. Impact of aortic stiffness on survival in end-stage renal disease. Circulation 1999;99:2434-9.

[17] Arnett DK, Boland LL, Evans GW, et al. Hypertension and arterial stiffness: the Atherosclerosis Risk in Communities Study. ARIC Investigators. Am J Hypertens 2000;13:317-23.

[18] Wilkinson IB, Qasem A, McEniery CM, et al. Nitric oxide regulates local arteria distensibility in vivo. Circulation 2002;105:213-7.

[19] Wilkinson IB, MacCallum H, Cockcroft JR, Webb DJ. Inhibition of basal nitric oxide synthesis increases aortic augmentation index and velocity in vivo. $\mathrm{Br} \mathrm{J}$ Clin Pharmacol 2002;53:189-92.

[20] Kinlay S, Creager MA, Fukumoto M, et al. Endothelium-derived nitric oxide regulates arterial elasticity in human arteries in vivo. Hypertension 2001;38:1049-53.

[21] Hashimoto H, Kitagawa K, Hougaku H, Etani H, Hori M. Relationship between $\mathrm{C}$-reactive protein and progression of early carotid atherosclerosis in hypertensive subjects. Stroke 2004;35:1625-30.

[22] Mahmud A, Feely J. Arterial stiffness is related to systemic inflammation in essential hypertension. Hypertension 2005;46:1118-22.

[23] De Silva DA, Woon FP, Gan HY, et al. Arterial stiffness, metabolic syndrome and inflammation amongst Asian ischaemic stroke patients. Eur J Neurol 2008;15(August (8)):872.

[24] Adams HP, Bendixen BH, Kappelle J, et al. Classification of subtype of acute ischemic stroke. Stroke 1993;e24:358.

[25] Wilkinson IB, MacCallum H, Flint L, et al. The influence of heart rate on augmentation index and central arterial pressure in humans. J Physiol 2000;525:263-7.

[26] Laurent S, Boutouyrie P, Asmar R, et al. Aortic stiffness is an independen predictor of all-cause and cardiovascular mortality in hypertensive patients. Hypertension 2001;37:1236-41.
[27] Guerin AP, Blacher J, Pannier B, et al. Impact of aortic stiffness attenuation on survival of patients in end-stage renal failure. Circulation 2001;103:98792.

28] Gow BS. The influence of vascular smooth muscle on the viscoelastic properties of blood vessels. In: Bergel DH, editor. Cardiovascular fluid dynamics. London: Academic Press; 1972. p. 66-97.

[29] Levy BI, Benessiano J, Poitevin P, Safar ME. Endothelium-dependent mechanical properties of the carotid artery in WKY and SHR: role of angiotensin converting enzyme inhibition. Circ Res 1990;66:321-8.

[30] Boutouyrie P, Bezie Y, Lacolley P, et al. In vivo/in vitro comparison of rat abdominal aorta wall viscosity: influence of endothelial function. Arterioscler Thromb Vasc Biol 1997; 17:1346-55.

[31] Hingorani AD, Cross J, Kharbanda RK, et al. Acute systemic inflammation impairs endothelium-dependent dilatation in humans. Circulation 2000;102:994-9.

[32] Bhagat K, Hingorani AD, Palacios M, Charles IG, Vallance P. Cytokine-induced venodilatation in humans in vivo: eNOS masquerading as iNOS. Cardiovasc Res 1999;41:754-64.

[33] Chia S, Qadan M, Newton R, et al. Intra-arterial tumor necrosis factor-alpha impairs endothelium-dependent vasodilatation and stimulates local tissue plasminogen activator release in humans. Arterioscler Thromb Vasc Biol 2003;23:695-701.

[34] Bassuk SS, Rifai N, Ridker PM. High sensitivity C-reactive protein: clinical importance. Curr Probl Cardiol 2004;29:439-93.

[35] Wilkinson IB, MacCallum H, Cockcroft JR, Webb DJ. Inhibition of basal nitric oxide synthesis increases aortic augmentation index and pulse wave velocity in vivo. Br J Clin Pharmacol 2002;53:189-92.

[36] Yip HK, Chen SS, Liu JS, et al. Serial changes in platelet activation in patients after ischemic stroke: role of pharmacodynamic modulation. Stroke 2004;35:1683-7.

[37] Chauhan AK, Kisucka J, Brill A, et al. ADAMTS13: a new link between thrombosis and inflammation. J Exp Med 2008;205(September (9)):206574.

[38] Licata G, Tuttolomondo A, Di Raimondo D, et al. Immuno-inflammatory activation in acute cardio-embolic strokes in comparison with other subtypes of ischaemic stroke. Thromb Haemost 2009;101(May (5)):929-37.

[39] Intengan HD, Schiffrin EL. Vascular remodeling in hypertension: roles of apoptosis, inflammation, and fibrosis. Hypertension 2001;38:581-7.

[40] Brown NJ. Aldosterone and vascular inflammation. Hypertension 2008;51:161-7.

[41] Abramson JL, Weintraub WS, Vaccarino V. Association between pulse pressure and C-reactive protein among apparently healthy US adults. Hypertension 2002;39:197-202.

[42] Park S, Kim JB, Shim CY, et al. The influence of serum aldosterone and the aldosterone-renin ratio on pulse wave velocity in hypertensive patients. J Hypertens 2007;25:1279-83.

[43] Mattace-Raso FU, van der Cammen TJ, van der Meer I, et al. C-reactive protein and arterial stiffness in older adults: the Rotterdam Study. Atherosclerosis 2004;176:111-6.

[44] Schnabel R, Larson MG, Dupuis J, Lunetta KL, Lipinska I, Meigs JB, Yin X, Rong J, Vita JA, Newton-Cheh C, Levy D, Keaney Jr JF, Vasan RS, Mitchell GF, Benjamin EJ. Relations of inflammatory biomarkers and common genetic variants with arterial stiffness and wave reflection. Hypertension 2008;51(June (6)):1651-7.

[45] Mitchell GF, Benjamin EJ. Relations of inflammatory biomarkers and common genetic variants with arterial stiffness and wave reflection. Hypertension 2008;51:1651-7.

[46] De Silva DA, Woon FP, Gan HY, et al. Arterial stiffness, metabolic syndrome and inflammation amongst Asian ischaemic stroke patients. Eur J Neurol 2008;15(August (8)):872-5.

[47] Lee SH, Choi S, Jung JH, Lee N. Effects of atrial fibrillation on arterial stiffness in patients with hypertension. Angiology 2008;59(August-September (4)):459-63.

[48] Samdani AF, Dawson TM, Dawson VL. Nitric oxide synthase in models of focal ischemia. Stroke 1997;28(June (6)):1283-8.

[49] Kaji Y, Miyoshi T, Doi M, et al. Augmentation index is associated with B-type natriuretic peptide in patients with paroxysmal atrial fibrillation. Hypertens Res 2009;32(July (7)):611-6. 